Incorporation of phosphorus donors in (110)-textured polycrystalline diamond Non Peer-reviewed author version

LAZEA, Andrada; Barjon, Julien; D'HAEN, Jan; MORTET, Vincent;

D'OLIESLAEGER, Marc \& HAENEN, Ken (2009) Incorporation of phosphorus donors in (110)-textured polycrystalline diamond. In: JOURNAL OF APPLIED PHYSICS, 105(8), (ART No 083545).

DOI: $10.1063 / 1.3116736$

Handle: http://hdl.handle.net/1942/9836 


\title{
Incorporation of phosphorus donors in (110)-textured
}

\section{polycrystalline diamond}

\author{
A. Lazea ${ }^{1,2}$, J. Barjon ${ }^{3}$, J. D’Haen ${ }^{1,2}$, V. Mortet ${ }^{1,2}$, M. D’Olieslaeger ${ }^{1,2}$, K. Haenen ${ }^{1,2, *}$ \\ ${ }^{1}$ Hasselt University, Institute for Materials Research (IMO), \\ Wetenschapspark 1, B-3590 Diepenbeek, Belgium \\ ${ }^{2}$ IMEC vzw, Division IMOMEC, Wetenschapspark 1, B-3590 Diepenbeek, Belgium \\ ${ }^{3}$ Groupe d'Etude de la Matière Condensée (GEMaC), Université de Versailles St Quentin - \\ CNRS, 1 place Aristide Briand, F-92195 Meudon cedex, France
}

\begin{abstract}
The incorporation efficiency of phosphorus was studied as a function of the surface orientation of grains in (110)-textured polycrystalline CVD diamond. Cathodoluminescence mapping of such films exhibits large local differences in relative intensities stemming from P-bound and free excitons. Combined with electron backscattering diffraction mapping, these data allow assessing of the donor concentration as a function of the grain orientation. While $[\mathrm{P}]$ can vary between $10^{15}$ and $>10^{18} \mathrm{~cm}^{-3}$ within one film, misorientation angles of more than $10^{\circ}$ with respect to the exact [110] axis assure an enhanced incorporation of $\mathrm{P}$ with concentrations surpassing $5 \times 10^{17} \mathrm{~cm}^{-3}$. The role of the surface morphology in the observation of these large incorporation differences is explained.
\end{abstract}

\footnotetext{
*ken.haenen@uhasselt.be
} 


\section{INTRODUCTION}

In order to use diamond as a semiconductor for the fabrication of electronic devices, understanding and controlling its electrical properties by doping is crucial. The range of impurities which satisfies the requests is limited, especially for n-type conduction. However, the last decade brought serious progress in this direction and nowadays phosphorus growth of $(001)$ or (111)-orientated diamond is relatively easy achieved by microwave plasma-enhanced chemical

vapour deposition (MW PE CVD). ${ }^{1-4}$ Nevertheless, the large difference in P-doping efficiency and compensation ratio in these two cases, show that the substrate orientation plays a significant role that is not fully understood to date. Recent results have shown that it is possible to grow a Pdoped microcrystalline form of (110)-oriented diamond using atypical growth conditions when compared to the growth parameters found in literature. ${ }^{5}$ To further elucidate the role of the substrate orientation on the P-incorporation efficiency in separate grains, electron back-scattered diffraction (EBSD) and cathodoluminescence (CL) mapping of (110)-textured P-doped diamond films were compared, yielding spatial information on the phosphorus concentration $[\mathrm{P}]$ according to the grain orientation. It will be shown that phosphorous is successfully incorporated in all the grains of the films although a certain off-angle with respect to the exact [110] direction is beneficial to enhance the P-incorporation.

\section{EXPERIMENTAL DETAILS}

For this study, the P-doped diamond layers were grown on polished undoped microcrystalline CVD diamond discs of $3 \mathrm{~mm}$ diameter. These discs were laser cut from a freestanding 2 inch diameter undoped microcrystalline diamond wafer and subsequently polished to yield smooth substrates for the P-doping experiments. Each disc was about $1.2 \mathrm{~mm}$ thick, showing grain sizes of several tens to hundreds of $\mu \mathrm{m}$. Although the orientation of all these substrate grains is clearly $\{110\}$ - 
oriented, all grains show a certain misorientation angle with respect to the ideal [110] direction as determined with electron backscattering diffraction (EBSD). ${ }^{5}$ Phosphorus doping was carried out using microwave plasma enhanced (MW PE CVD) in an ASTeX PDS 17 steel chamber type apparatus. Phosphine $\left(\mathrm{PH}_{3}\right)$ was used as the phosphorus source and added to the conventional methane-hydrogen mixture. The growth temperature was monitored using a disappearing filament pyrometer and maintained around $1140{ }^{\circ} \mathrm{C}$, while the $\left[\mathrm{CH}_{4}\right] /\left[\mathrm{H}_{2}\right]$ ratio was equal to $1 \%$. The P-doped layers were grown during $2 \mathrm{~h}$, leading to a P-doped film thickness of about $20 \mu \mathrm{m}$ with grain size similar to the ones of the substrate. Detailed explanations of the procedure and conditions for the sample preparation are described elsewhere. ${ }^{5}$ Morphology and structure of the films were studied with automated electron backscattering diffraction (EBSD) using a HHL system installed in a scanning electron microscope (SEM) type Quanta 200-FEG manufactured by FEI. The typical penetration depth of the EBSD e-beam is 10 to $50 \mathrm{~nm}$ yielding the surface orientation of the different grains, which is $\{110\}$-textured. Cathodoluminescence (CL) spectroscopy and mapping at $102 \mathrm{~K}$ were performed with a Horiba Jobin Yvon SA system installed on a JEOL840 scanning electron microscope. The P-doped diamond layer was excited with a $20 \mathrm{keV}$ focussed electron beam (corresponding to a penetration depth of $\sim 2.8 \mu \mathrm{m}$ ) at a $0.2 \mu \mathrm{A}$ current. The epitaxial layers were coated with a semi-transparent gold layer $(\sim 5 \mathrm{~nm})$ in order to evacuate electric charges. The CL emission was collected by a parabolic mirror and injected with metal optics into a monochromator equipped with a UV enhanced-silicon CCD camera and a 600 grooves/mm diffraction grating. The CL maps were obtained by acquiring the CL spectra of $64 \times 64$ points scanning different grains inside a 380x300 $\mu \mathrm{m}^{2}$ window.

\section{RESULTS AND DISCUSSION}

As discussed in ref. ${ }^{6}$ and ${ }^{7}$ by Barjon et al., it is possible to determine the active P-concentration in CVD diamond by making use of the ratio of the free and P-bound exciton luminescence intensities. Hence, it is necessary that both types of exciton luminescence are detectable in the CL 
spectra to make a successful estimation. However, when investigating films grown with $500 \mathrm{ppm}$ $\left[\mathrm{PH}_{3}\right] /\left[\mathrm{CH}_{4}\right]$ in the gas phase, nearly all the exciton-related emission is attributed to the P-bound exciton, and this for different spots on the film, i.e. different oriented grains. The absence of free exciton emission points to an overall $[\mathrm{P}]$ that is higher than $10^{18} \mathrm{~cm}^{-3}$, i.e. the phosphorus donor is efficiently incorporated using the applied growth conditions.

Figure 1 shows the CL spectra taken on 2 different grains from a film grown using less phosphorus in the gas phase, with $100 \mathrm{ppm}\left[\mathrm{PH}_{3}\right] /\left[\mathrm{CH}_{4}\right]$. The two grains, which are in close proximity, and are marked accordingly in Figure 2(a), both fulfil the conditions enabling the quantification of the P-concentration by CL. The spectra shows distinguishable peaks which can be attributed to free (FE) and P-bound $\left(\mathrm{BE}_{\mathrm{P}}\right)$ excitons, labelled TO or TA when assisted with a transverse optical or acoustic phonon respectively and NP in the case of a no phonon transition. ${ }^{6-9}$ $\mathrm{O}^{\Gamma}$ is the phonon involved in optical replica. Although both grains show several types of exciton emission, including the $\mathrm{FE}^{\mathrm{TO}}$ and $\mathrm{BE}_{\mathrm{P}}^{\mathrm{TO}}$ signals used for $[\mathrm{P}]$ quantification, it is clear that there is a large difference in their intensity and position. In grain 1 the spectrum is dominated by free exciton recombination. In contrast, the situation for grain 2 is clearly different. The bound exciton related emission is much larger than the signal related to free excitons - it indicates a larger incorporation of substitutional phosphorus in grain 2. In addition; the energetic positions of all exciton recombinations are shifted towards lower energies in grain 2. Such a lowering in energy has already been reported in the case of polycrystalline P-doped films deposited directly on silicon, pointing to a large position dependent tensile stress, and, as a result, a local bandgap reduction. ${ }^{10}$ The presence of strain inside grains is also coherent with the large $\mathrm{BE}_{\mathrm{P}}{ }^{\mathrm{TO}}$ linewidths 
(43 meV) we observed. Such linewidths are well above those obtained for homoepitaxial layers $(23 \mathrm{meV}) .{ }^{6,7}$

The key point of immediate interest is the difference in the ratio of $\mathrm{FE}^{\mathrm{TO}}$ and $\mathrm{BE}_{\mathrm{P}}{ }^{\mathrm{TO}}$ emission for grain 1 and 2 indicating that the amount of incorporated $\mathrm{P}$ is clearly different for both grains, with a clear enhanced incorporation of $\mathrm{P}$ in grain 2 where $[\mathrm{P}]>10^{17} \mathrm{~cm}^{-3}$. To study the incorporation in the film, the whole surface was mapped by $\mathrm{CL}$ and $[\mathrm{P}]$ was determined. Figure 2(a) shows a typical result of a $380 \times 300 \mu \mathrm{m}^{2}$ area. The CL mapping confirms that there is a large variation of the phosphorus concentration from one grain to another, with values ranging between $10^{15}$ and $10^{18} \mathrm{~cm}^{-3}$. To correlate these results with the grain orientation, a corresponding EBDS map was made showing the misorientation angle $\theta_{\text {off }}$ [110] of the grains with respect to the perfect [110] direction. All grains fall within the $4^{\circ}$ to $20^{\circ}$ interval. Comparing both figures, it is remarkable that the P-incorporation efficiency is smaller for grains oriented closer to the [110] direction, while there is systematically a large amount of phosphorus donor atoms incorporated when these angles are higher than $12^{\circ}$. The relation between the orientation and the donor concentration is plotted in Figure 3. While the influence of the misorientation is obvious, the lack of a clear oneto-one relation between the two quantities $[\mathrm{P}]$ and $\theta_{\text {off }[110]}$, leads to the assumption that there is at least a second element that plays a key role. The influence of the substrate misorientation angle was studied before for single crystal n-type (100)-oriented diamond layers, where it was found that small angles of $2^{\circ}$ or $3^{\circ}$ were useful in enhancing step flow growth and the phosphorus incorporation. ${ }^{3}$ The beneficial influence was explained by the need to control the step growth and thus to reduce the presence of the non-epitaxial crystallites and pyramidal hillocks. Nevertheless, the amount of phosphine gas needed to obtain similar $[\mathrm{P}]$ as presented here, was two orders of 
magnitude higher. When looking in detail to the surface of grain 2 (Figure 2(c)), it becomes obvious that a certain roughness is present, which is also evident in the granular-like parts of Figure 2(b). It is proposed that the surface features consist out of (111)-oriented microfacets that facilitate the incorporation of P. Studies on single crystal diamond have already pointed out that the incorporation efficiency of (111)-oriented layers is several orders of magnitude higher than for (100)-oriented films. ${ }^{3}$ Moreover, van Enckevort and co-workers have shown that growth on top of (110) faces is stabilised through the formation of microfacets, leading to the rough appearance as seen in Figure 2(c). ${ }^{11}$ According to Nishitani-Gamo et al. these microfacets develop predominantly in (111) facets. ${ }^{12}$ In addition, calculations in ref. 13 lead to believe that a certain misorientation with respect to the exact [110] axis can have an advantageous effect on the growth rate, enhancing locally the growth and subsequently the phosphorus incorporation of suitable oriented grains. Contrary to this, grains with a misorientation angle lower than $10^{\circ}$ show much less to no microfacets at all, maintaining their $\{110\}$ orientation. As a result, these regions generally have an a $[\mathrm{P}]$ below $10^{16} \mathrm{~cm}^{-3}$. Examples can be seen in the bottom left and top right corners of Figure 2(a),

\section{CONCLUSION}

In summary, a detailed investigation of n-type doping of (110)-oriented microcrystalline diamond was achieved by CL and EBSD mapping. We have proven that the combination of these techniques is a powerful tool in the local determination of dopant incorporation as a function of the substrate orientation. Such data are essential to optimize the P-doping of single and polycrystalline CVD diamond layers. In particular, it was shown that the phosphorus concentration in one film can vary over three orders of magnitude, mainly determined by the 
misorientation angle of the grain with respect to the perfect [110] axis. Whereas a low misorientation angle leads to a low $\mathrm{P}$ concentration, angles of more than $10^{\circ}$ with respect to the perfect [110] axis are needed to incorporate more than $10^{17} \mathrm{~cm}^{-3}$. For the latter, we propose that the surface morphology facilitates the local incorporation of $\mathrm{P}$ via the formation of (111)-oriented microfacets. 


\section{ACKNOWLEDGMENTS}

The authors want to express their gratitude to prof. Nesládek (Hasselt University), dr. M. Schreck (Universität Augsburg) and prof. J. Achard (CNRS-LIMHP) for fruitful discussions. This work was financially supported by the EU FP6 Marie Curie RTN "DRIVE" (MRTN-CT-2004512224), the Research Programs G.0068.07 and G.0430.07 of the Research FoundationFlanders (FWO), the Methusalem "NANO network", and the IAP-P6/42 project 'Quantum Effects in Clusters and Nanowires'. 


\section{References}

1 S. Koizumi, M. Kamo, Y. Sato, H. Ozaki, T. Inuzuka, Appl. Phys. Lett. 71, 1065 (1997).

2 M. Nesladek, Semicond. Sci. Technol. 20, R19 (2005).

3 H. Kato, T. Makino, S. Yamasaki, H. Okushi, J. Phys. D-Appl. Phys. 40, 6189 (2007).

4 M.-A. Pinault, J. Barjon, T. Kociniewki, F. Jomard, J. Chevallier, Physica B. 401-402, 51 (2007).

5 A. Lazea, V. Mortet, J. D’Haen, P. Geithner, J. Ristein, M. D’Olieslaeger, K. Haenen, Chem. Phys. Lett. 454, 310 (2008).

6 J. Barjon, P. Desfonds, M.-A. Pinault, T. Kociniewski, F. Jomard, J. Chevallier, J. Appl. Phys. 101, 113701 (2007).

7 J. Barjon, M.-A. Pinault, T. Kociniewski, F. Jomard, J. Chevallier, Phys. Stat. Sol. A 209, 2965 (2007).

8 R. Sauer, N. Teofilov, K. Thonke, S. Koizumi, Phys. Stat. Sol. A 201, 2405 (2004).

9 D. Araujo, A. Tajani, E. Gheeraert, E. Bustarret, J. Phys.: Condens. Matter. 16, S287 (2004).

${ }^{10}$ S. Ghodbane, F. Omnès, E. Bustarret, C. Tavares, F. Jomard, Diamond Relat. Mater 17, 1324 (2008).

${ }^{11}$ W.J.P. van Enckevort, G. Janssen, W. Vollenberg, J.J. Schermer, L.J. Giling, M. Seal, Diamond Relat. Mater. 2, 997 (1993).

${ }^{12}$ M. Nishitani-Gamo, K.P. Loh, I. Sakaguchi, T. Takami, I. Kusunoki, T. Ando, J. Vac. Sci. Technol. A 17, 2991 (1999).

${ }^{13}$ C.C. Battaile, D.J. Srolovitz, J.E. Butler, J. Cryst. Growth 194, 353 (1998). 
Figure 1. (Color online) Cathodoluminescence spectra taken at $\sim 100 \mathrm{~K}$ on two different oriented grains of a (110)-textured $100 \mathrm{ppm}\left[\mathrm{PH}_{3}\right] /\left[\mathrm{CH}_{4}\right]$ doped film (see Figure 2). Grain 2 shows more P-bound exciton emission than grain 1, pointing to a higher incorporation of the substitutional $\mathrm{P}$ donor.

Figure 2. (Color online) a) P-concentration map based on the ratio of intensities of neutral phosphorus-bound exciton and free exciton emission as determined with CL mapping. "1" and "2" mark the two spots where the CL spectra featured in Figure 1 were taken. b) Corresponding EBSD map showing the misorientation angle of the grains with respect to the exact [110] direction. The colour legend corresponds to off-angles between 0 and 20 degree, with a resolution of 2 degrees. c) SEM picture of the region featured in a) and b).

Figure 3. (Color online) Phosphorus concentration in function of the off-angle of the grain surface orientation with respect to the perfect [110] axis. 


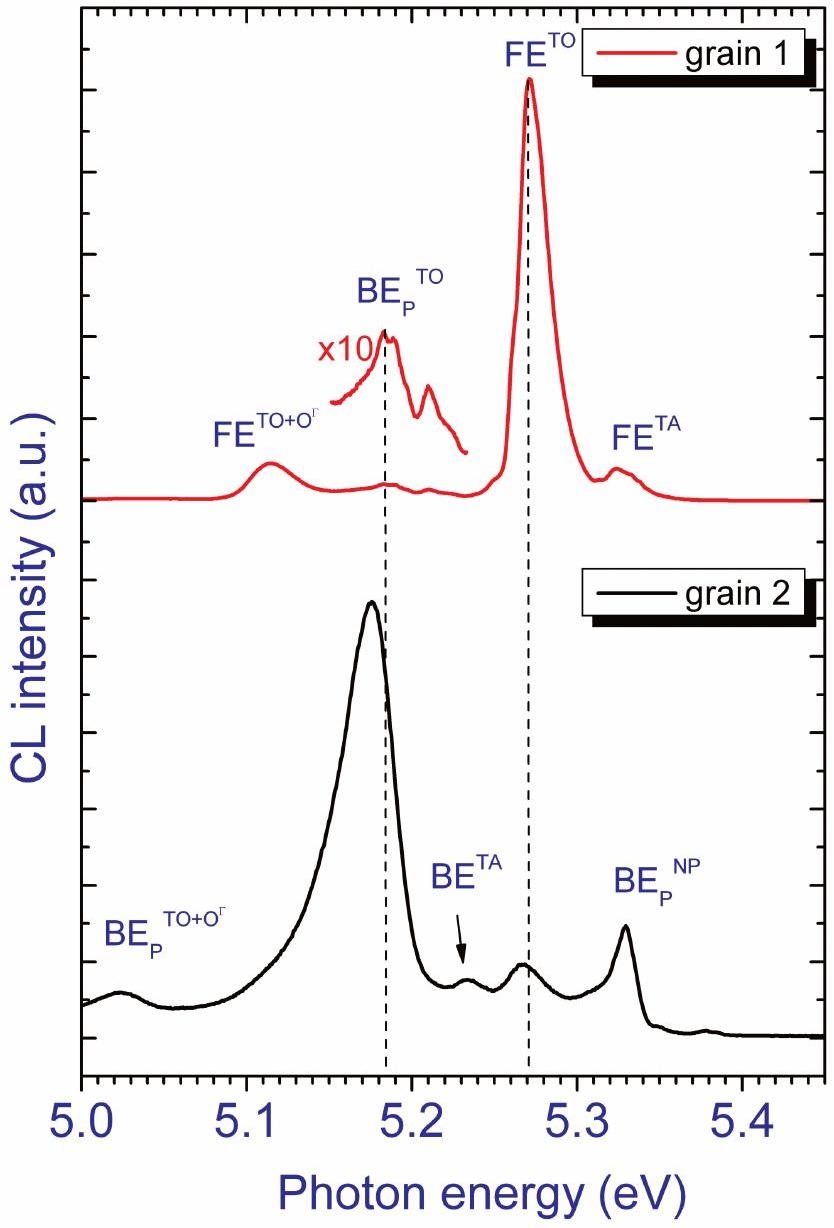


a) $\mathrm{CL}$

2

Phosphorus donor concentration $\left(\mathrm{cm}^{-3}\right)$ 


$$
\text { b) EBSD }
$$

(-)

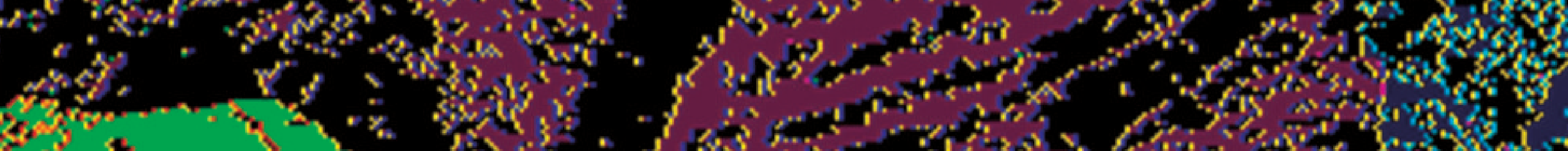
4 (n)

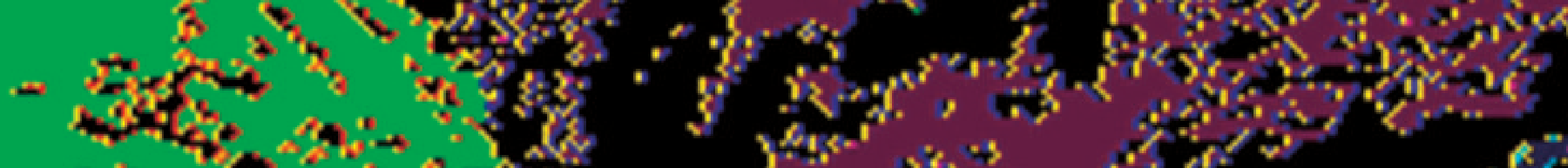

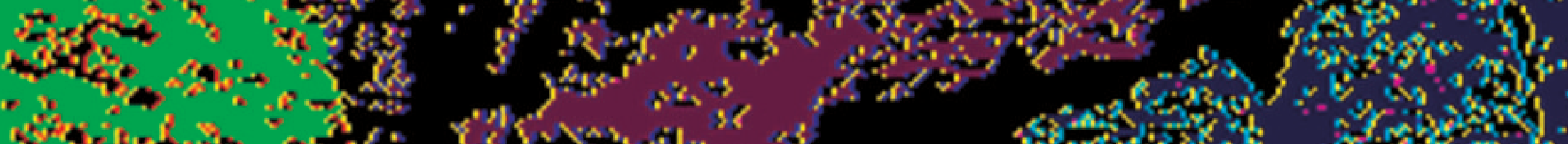
or an an

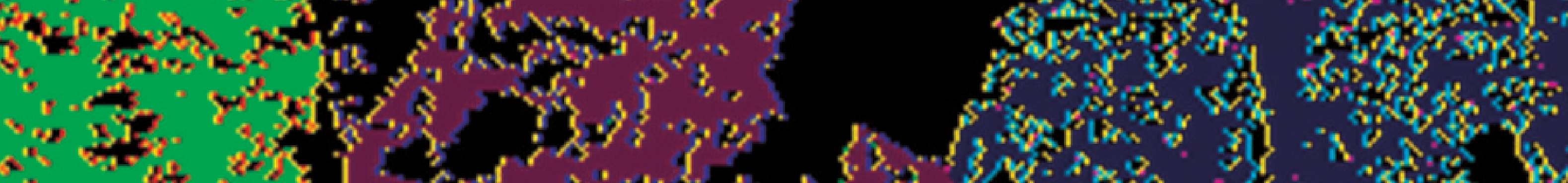

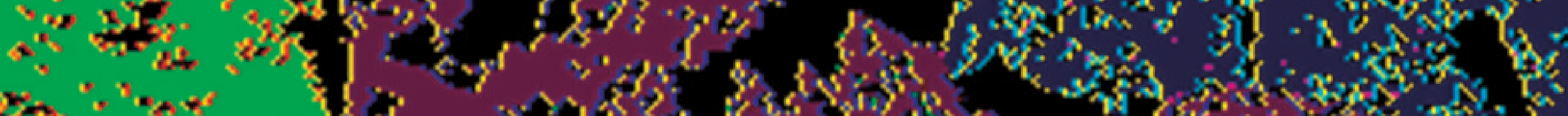

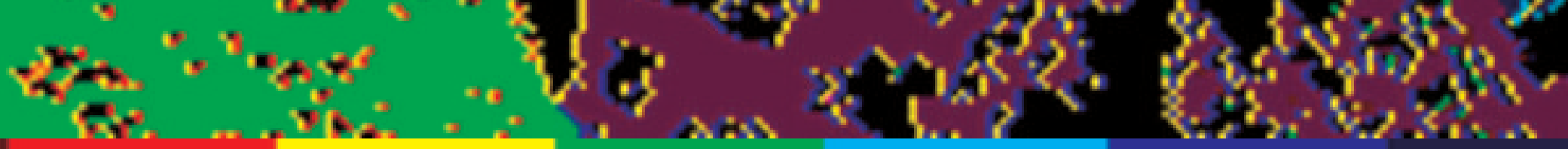
(1),

\section{$\theta_{\text {off [110] }}\left({ }^{\circ}\right)$}


c) SEM
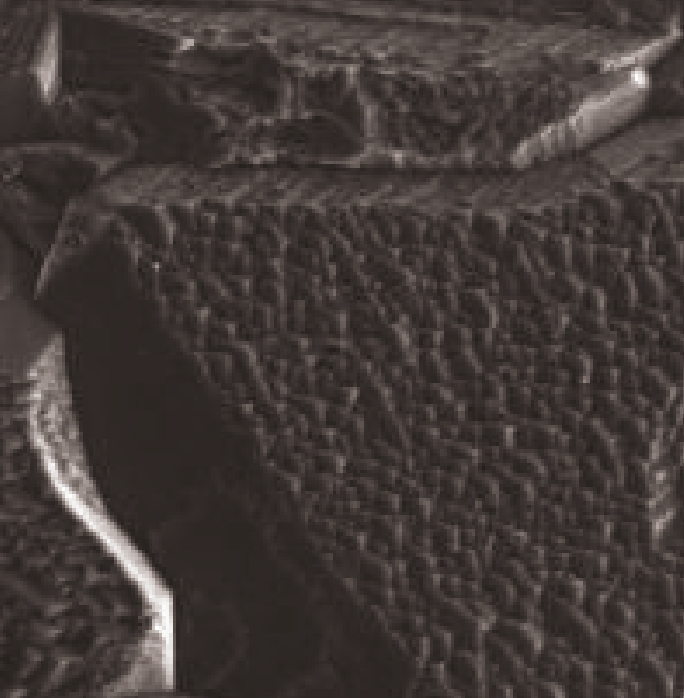

1
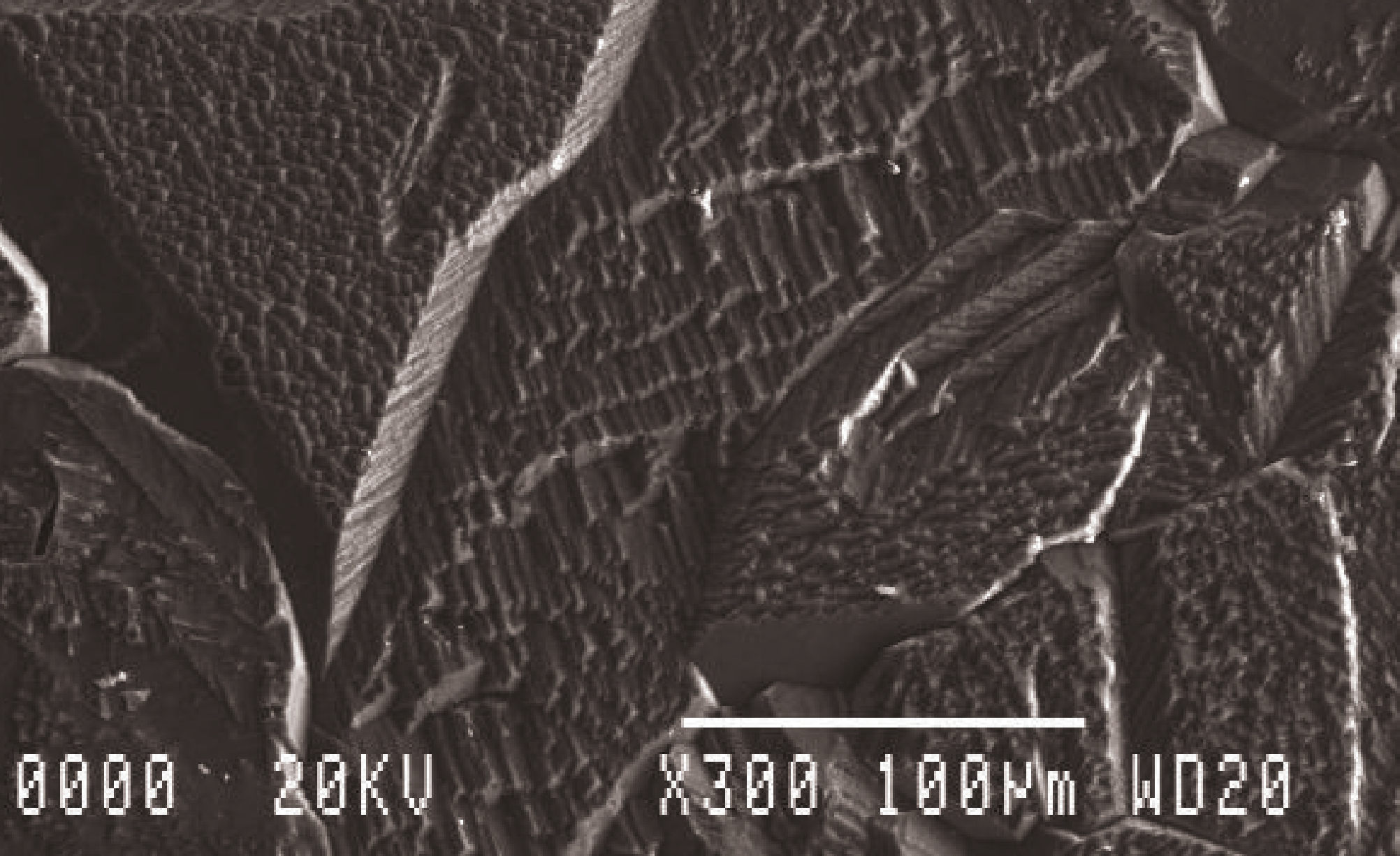


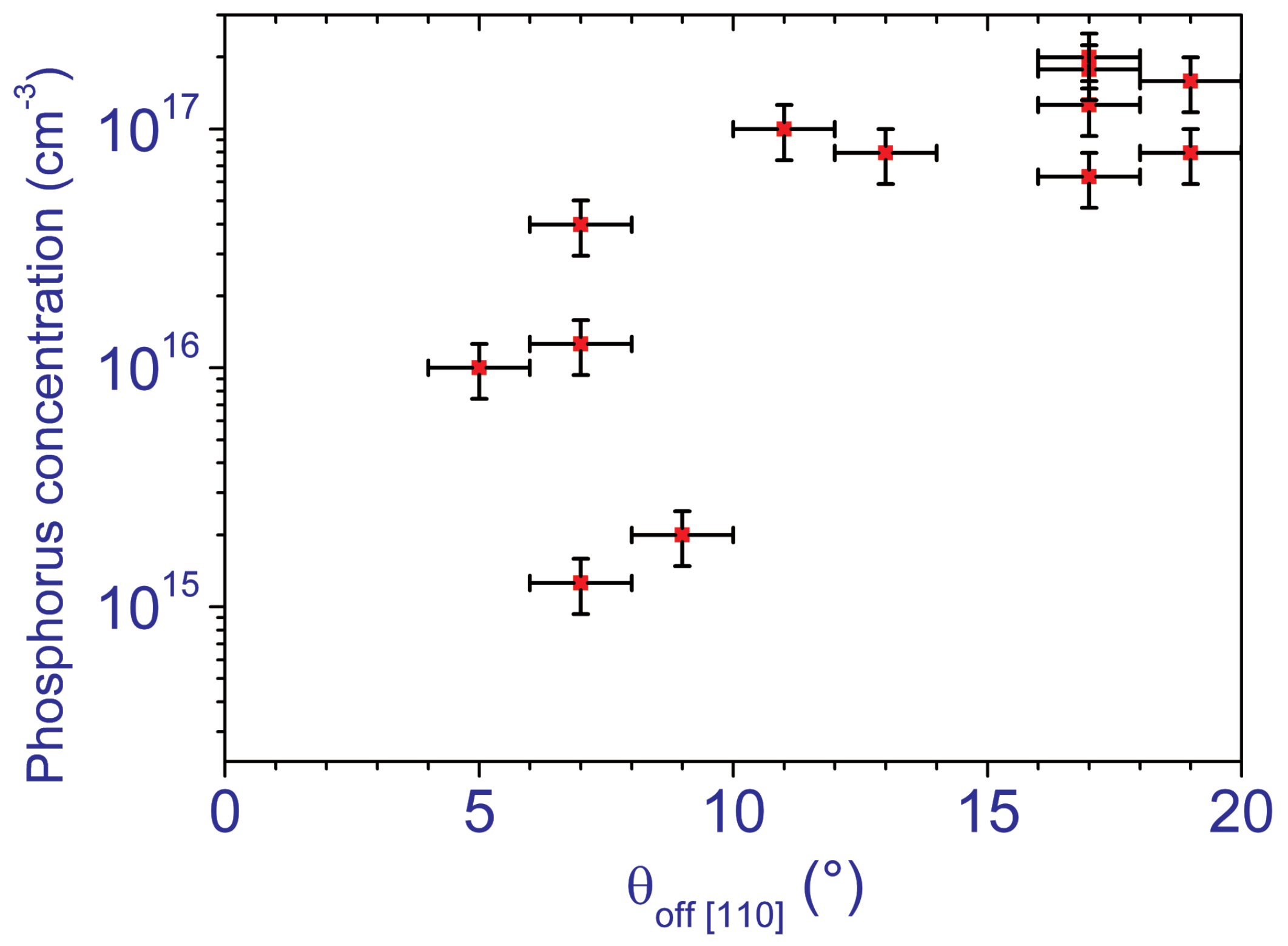

